\title{
NAD-Dependent Deacetylase Sirtuin-3, Mitochondrial
}

National Cancer Institute

\section{Source}

National Cancer Institute. NAD-Dependent Deacetylase Sirtuin-3, Mitochondrial. NCI

Thesaurus. Code C95591.

NAD-dependent deacetylase sirtuin-3, mitochondrial (399 aa, $44 \mathrm{kDa}$ ) is encoded by the human SIRT 3 gene. This protein plays a role in the deacetylation of proteins. 